Acta Zoológica Mexicana (nueva serie), Volumen 37, 1-17.

https://doi.org/10.21829/azm.2021.3712379

Artículo original

\title{
Odonata (Insecta) como indicador de la calidad ambiental en humedales de montaña neotropicales
}

\section{Odonata (Insecta) as an indicator of environmental quality in neotropical mountain wetlands}

\section{(D) 1*JUAN ANTONIO LÓPEZ-DÍAZ, (D) 23ENIGNO GÓMEZ, (D) ${ }^{3}$ ENRIQUE GONZÁLEZ-SORIANO, (D) 'MARÍA GÓMEZ-TOLOSA}

${ }^{1}$ Universidad de Ciencias y Artes de Chiapas, Instituto de Ciencias Biológicas. Libramiento Norte-Poniente s/n, Col. Lajas Maciel. Tuxtla Gutiérrez, Chiapas, México. C.P. 29000.

${ }^{2}$ El Colegio de la Frontera Sur, Departamento de Conservación de la Biodiversidad. Carretera Panamericana y Periférico Sur s/n, Barrio de María Auxiliadora, San Cristóbal de Las Casas, Chiapas, México. C.P. 29290. ${ }^{3}$ Universidad Nacional Autónoma de México, Instituto de Biología, Apartado postal 70-153. México, CDMX, C.P. 04510.

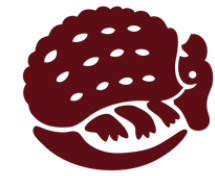

OPEN ACCESS

*Autor corresponsal:

Juan Antonio López Díaz juanlodi44@gmail.com

Cita:

López-Díaz, J. A., Gómez, B., González-Soriano, E., GómezTolosa, M. (2021) Odonata (Insecta) como indicador de la calidad ambiental en humedales de montaña neotropicales. Acta Zoológica Mexicana (nueva serie),

$37,1-17$.

10.21829/azm.2021.3712379 elocation-id: e3712379

Recibido: 08 diciembre 2020 Aceptado: 25 mayo 2021 Publicado: 22 junio 2021
Editor responsable: Magdalena Cruz Rosales

RESUMEN. Con el fin de evaluar la diversidad del orden Odonata y relacionarla con la calidad del ambiente, en San Cristóbal de Las Casas (Chiapas) se eligieron cuatro humedales de acuerdo con su accesibilidad y permisibilidad. Para la tipología ambiental se consideró, entre otras características, la calidad visual, estimada a través del Índice del Estado de Conservación de Humedales (IECH). Recolectas sistemáticas mensuales de los odonatos adultos fueron realizadas entre julio de 2014 y junio de 2015. Un total de 14 especies, distribuidas en cinco familias y 10 géneros fueron encontradas. El género Argia fue el de mayor riqueza, mientras que Ischnura denticollis y Enallagma rua representaron las especies dominantes. Las Cañadas, humedal con calidad visual subóptima, presentó la mayor diversidad de libélulas al integrar un ambiente lótico. El grado de alteración de la vegetación acuática, como uno de los parámetros evaluados en la calidad visual, fue muy importante en las diferencias encontradas en la diversidad y abundancia de Odonata en los cuatro humedales. María Eugenia y La Kisst 
catalogados como ambientes con calidad visual óptima y regular, respectivamente, obtuvieron la mayor similitud en su odonatofauna relacionada con las distintas formas de vida de su vegetación acuática y por el tamaño equivalente de estos dos humedales.

Palabras clave: libélulas; diversidad; Ramsar; calidad visual

ABSTRACT. In order to evaluate the diversity of the Odonata order and relate it to environmental quality, in San Cristobal de Las Casas (Chiapas) four wetlands were chosen according to their accessibility and permissibility. For the environmental typology, among other characteristics, the visual quality was considered, estimated through the Index of the State of Conservation of Wetlands (IECH). Systematic monthly collections of adult odonates were carried out between July 2014 and June 2015. A total of 14 species, distributed in five families and 10 genera were found. The genus Argia was the richest, while Ischnura denticollis and Enallagma rua represented the dominant species. Las Cañadas, a wetland with suboptimal visual quality, presented the greatest diversity of dragonflies by integrating a lotic environment. As one of the parameters evaluated in the visual quality, the degree of alteration of the aquatic vegetation was very important in the differences found in the diversity and abundance of Odonata in the four wetlands. Maria Eugenia and La Kisst classified as environments with optimal and regular visual quality, respectively, obtained the greatest similarity in their odonatofauna related to the different life forms of their aquatic vegetation and for the equivalent size of these two wetlands.

Key words: dragonflies; diversity; Ramsar; visual quality

\section{INTRODUCCIÓN}

La urbanización es un proceso continuo cuyos efectos sobre la diversidad biológica están fuertemente relacionados a los atributos de los ecosistemas en distintas escalas espaciales (McDonell \& Pickett, 1990). Estos efectos son especialmente fuertes en los ecosistemas de humedal, que a pesar del reconocimiento generalizado de que estos ambientes brindan valiosos servicios ecológicos (Woodward \& Wui, 2001), la escasez de áreas para el desarrollo urbano ha conllevado a la destrucción de humedales alrededor del mundo (Holland et al., 1995).

San Cristóbal de Las Casas (Chiapas, México) cuenta con humedales de montaña que mantienen la alimentación y recarga de mantos freáticos, proveen el $70 \%$ del agua entubada para consumo humano que se distribuye hacia la ciudad y constituyen un área de distribución de especies endémicas (SEMAHN, 2011). En 1528, San Cristóbal contaba con 2,500 hectáreas de superficie de humedales, mientras que para el 2006 se registraban tan sólo 106 hectáreas (4.24\%) (Vásquez, 2007). En este caso, el proceso de urbanización ha conducido a que estos ecosistemas se encuentren actualmente en el interior de la mancha urbana, exponiéndolos a un gran deterioro y llevándolos a una disminución sustancial del agua y de sus comunidades biológicas (Cruz \& Hernández, 2010).

Entre las comunidades de invertebrados que habitan en los humedales se destacan las pertenecientes al orden Odonata (Insecta), el cual integra a un grupo de insectos acuáticos 
comúnmente llamados libélulas (suborden Anisoptera) y caballitos del diablo (suborden Zygoptera), que a nivel mundial consta de 6,322 especies (Schorr \& Paulson, 2019). En México se tienen registradas 356 especies de odonatos (Cuevas-Yañez et al., 2017b), de las cuales 187 están catalogadas para el estado de Chiapas; de estas, 186 especies fueron documentadas por GonzálezSoriano y Paulson (2011) basados en la información de 171 localidades (11 especies distribuidas en San Cristóbal), y a esto se adiciona el registro de Brachymesia herbida (Gundlach, 1889), recolectada en la región Soconusco del estado (Gómez-Tolosa et al., 2015).

Como larva o adulto, los odonatos tienen un papel fundamental dentro de las redes tróficas (Ramírez, 2010) y sus exigencias ecológicas les permiten ser considerados potenciales bioindicadores de la calidad ambiental al responder directa e indirectamente ante la perturbación natural (Stewart \& Samways, 1998) o antropogénica (Bulánková, 1997; Corbet, 1999; Catling, 2005; Chovanec \& Waringer, 2005; Benazzouz et al., 2009; Monteiro-Júnior et al., 2015; Cuevas-Yañez et al., 2017a; Worthen \& Chamlee, 2020). En este sentido, son diversos los estudios en los que se analizan los atributos de los ensambles de libélulas con relación a la integridad de su hábitat, tanto en ambientes lóticos (Chovanec \& Waringer, 2001; De Paiva et al., 2010; Gómez-Tolosa et al., 2015) como lénticos (Chovanec \& Waringer, 2005; Kutcher \& Bried, 2014). Por lo tanto, nuestra hipótesis de trabajo es demostrar que existen ensambles de Odonata asociados a humedales de mejor calidad ambiental, independientemente si pertenecen a un sistema lótico o léntico. El objetivo del presente estudio es analizar la diversidad de libélulas y caballitos del diablo adultos con la calidad ambiental visual en cuatro humedales de montaña en San Cristóbal de Las Casas; México.

\section{MATERIALES Y MÉTODOS}

Área de estudio. Comprendió cuatro humedales de montaña de zonas periurbanas, y considerando su accesibilidad y permisibilidad, fueron seleccionados aleatoriamente: Las Cañadas (LC), María Eugenia (ME), La Kisst (LK) y Cinco de Marzo (CM). Estos ambientes están localizados en el municipio de San Cristóbal de Las Casas (en adelante SCLC), Chiapas, con coordenadas $16^{\circ} 45^{\prime}$ Norte, $92^{\circ} 38^{\prime}$ Oeste, a 2,120 m s.n.m. (Fig. 1). El clima es templado húmedo con lluvias en verano y temperatura máxima anual promedio de $23^{\circ} \mathrm{C}$. Su vegetación está compuesta por bosque de pinoencino (SEMAHN, 2011).

Tipología ambiental. Los sitios fueron caracterizados de acuerdo con el tipo de sistema acuático (lótico / léntico), tamaño (estimado a través de Google Earth), decreto legal de conservación (para reconocer a través de la revisión de literatura actividades que promuevan la conservación de los humedales), diversidad de macrófitas y calidad visual. Parcelas aleatorias de un metro cuadrado con recolectas botánicas, que partieron desde la orilla hasta la parte más seca del humedal (Stewart \& Kantrud, 1971) fueron consideras para estimar la diversidad de macrófitas (índice de Shannon). La calidad visual integra un enfoque de observación rápida para la evaluación de la calidad del hábitat que apoya la condición biológica óptima (Barbour et al., 1999). El índice del estado de conservación ecológica de humedales (IECH, modificado de Barbour et al., 1999 y Boix et al., 2010) fue utilizado para determinar este atributo que valora las características físicas que influyen en la calidad del agua (infraestructura y usos humanos, aspecto del agua) y la condición de su vegetación asociada (composición de la comunidad vegetal y grado de alteración). 
Inventario faunístico. Fueron realizadas recolectas sistemáticas mensuales de julio de 2014 a junio de 2015. Cada humedal se recorrió en una sola ocasión, en un transecto de $1 \mathrm{~km}$ durante seis horas (08:00-14:00 horas) de recolecta de los adultos de Odonata con red entomológica aérea. El muestreo fue realizado por una persona (Cuevas-Yañez, 2007). Las muestras de los primeros seis meses no se regresaron al ambiente, para los meses restantes, las especies reconocidas fueron marcadas en el área no pigmentada en una de sus alas y liberadas en campo. Un bolígrafo permanente fue utilizado para asignar un número distintivo a cada individuo (Palacino-Rodríguez \& Contreras-Sánchez, 2014). Los odonatos recolectados se almacenaron en una hielera para disminuir su metabolismo.

Trabajo de laboratorio. Los insectos pasaron por un proceso de acetonización durante 24 horas para conservar su coloración (Morse, 1998). La identificación se realizó utilizando claves taxonómicas, además del cotejo de las especies reportadas en el estado de Chiapas de acuerdo con González-Soriano y Paulson (2011). Los ejemplares se depositaron en la colección entomológica de El Colegio de la Frontera Sur, unidad San Cristóbal (ECO-SC-E).

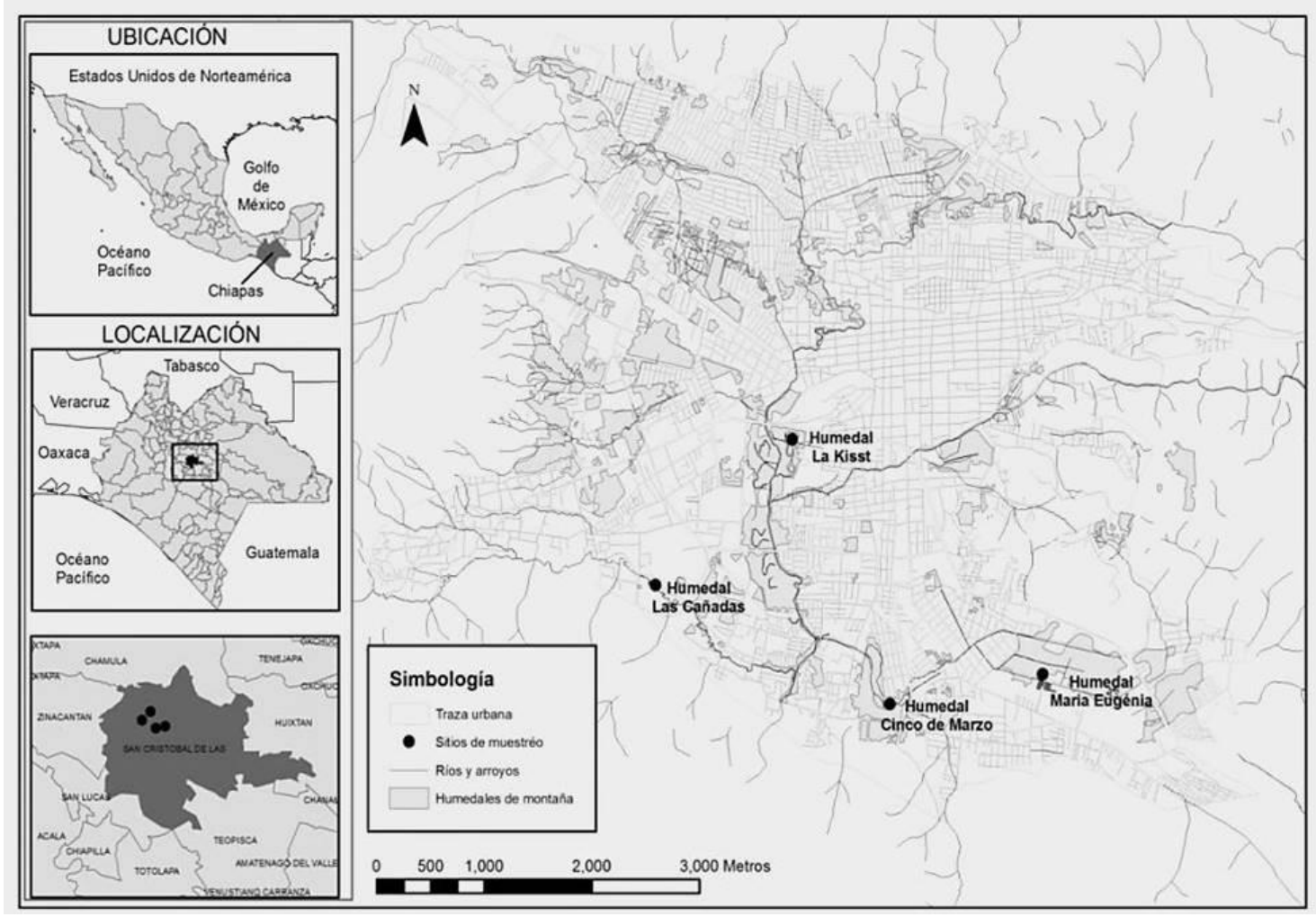

Figura 1. Localización geográfica de los humedales de montaña en San Cristóbal de Las Casas, Chiapas, México.

Análisis de datos. La riqueza de Odonata se consideró como el número de especies y la abundancia como el número de individuos por especie. La calidad del inventario se evaluó con una curva de acumulación de especies utilizando el estimador no paramétrico Chao 1 por medio 
de EstimateS 9.0 (Colwell, 2004). Para estimar las diferencias estadísticas en riqueza y abundancia de Odonata en los cuatro humedales, se evaluó, de manera preliminar, la normalidad y homocedasticidad de varianzas en estos datos con el programa Statistica 8.0 (StatSoft, 1991). Posteriormente, y debido a la normalidad de los datos, se realizó una prueba ANOVA únicamente para evaluar la riqueza de especies. Por otro lado, la prueba Kruskal-Wallis fue considerada para estimar diferencias en la abundancia de odonatos al presentar una distribución no normal.

Debido a que la mayoría de los índices de diversidad tradicionales priorizan la riqueza o la igualdad de las especies sin estimar ambos componentes de diversidad de forma simultánea (Tuomisto, 2010), es necesaria una medida estadística compuesta (Guisande et al., 2017). Para evitar este sesgo, utilizamos perfiles beta de diversidad (Di Battista et al., 2017) a través del paquete BioFT (Di Battista et al., 2016) con el software R (R Core Team, 2013), para ordenar a los humedales de mayor a menor diversidad. Di Battista et al. (2017) propusieron tres medidas funcionales para examinar el perfil de diversidad (la primera y segunda derivada, el radio de curvatura y la longitud de la curvatura) como una medida escalar para cada comunidad, conocida como el área bajo la curva.

El recambio de especies entre humedales fue examinado con el índice de similitud de Morisita-Horn. Usando el software Past 3.0 (Hammer et al., 2001) se realizó un análisis de agrupamiento por pares mediante ligamiento promedio no ponderado (UPGMA) para obtener un dendrograma de similitud faunística entre los diferentes humedales (Crisci \& López, 1983).

\section{RESULTADOS}

\section{Tipología ambiental}

ME y LK son los humedales de mayor tamaño, integran sistemas lénticos con tiempo perenne del agua y poseen la mayor diversidad de macrófitas (Cuadro 1), además, están sujetos a políticas de conservación de carácter nacional e internacional, en donde la reforestación y, para el caso de LK, la extracción del lirio acuático Eichornnia crassipes (C. Mart.) Solms, son actividades gestionadas en estos ambientes. Es importante mencionar que los cuatro humedales se encuentran perturbados, no obstante, ME y LK presentaron calidad visual óptima y regular, respectivamente. $\mathrm{CM}$ es el humedal con la menor área e integra un sistema léntico con tiempo perenne del agua, está inmerso en un asentamiento humano irregular y, por lo tanto, los residentes no cuentan con servicios de agua potable y saneamiento, conduciendo al relleno de su superficie inundable, la presencia de residuos sólidos urbanos e incendios de su vegetación acuática. Esta última está dominada por el junco Schoenoplectus californicus (C.A. Mey.) Soják, que es forrajeado por ganado vacuno. En este sentido, CM presentó la menor diversidad de macrófitas y una calidad visual pobre. Por su lado, LC representa un cuerpo de agua lótico estacional (abril-noviembre), conectado por un canal del arroyo que recibe el mismo nombre. Este ambiente cuenta con dos veces el tamaño de CM y posee alta diversidad de macrófitas, aunque menor en comparación a la de ME y LK, sin embargo, la ganadería afecta su vegetación de ribera y, por lo tanto, presentó una calidad visual subóptima. 
Cuadro 1. Tipología ambiental de cuatro humedales de montaña de San Cristóbal de las Casas, Chiapas, México.

\begin{tabular}{|c|c|c|c|c|c|c|}
\hline Humedal & $\begin{array}{l}\text { Tipo de } \\
\text { sistema }\end{array}$ & $\begin{array}{l}\text { Área } \\
\text { (Ha) }\end{array}$ & $\begin{array}{l}\text { Decreto legal } \\
\text { de } \\
\text { conservación }\end{array}$ & $\begin{array}{l}\text { Diversidad } \\
\text { de } \\
\text { macrófitas } \\
\left(\mathrm{H}^{\prime}\right) \\
\end{array}$ & $\begin{array}{l}\text { Impacto } \\
\text { antropogénico }\end{array}$ & $\begin{array}{l}\text { Calidad } \\
\text { visual }\end{array}$ \\
\hline LC & Lótico & 34.97 & & 2.468 & $\begin{array}{l}\text {-Contaminación } \\
\text { significativa por } \\
\text { residuos sólidos } \\
\text {-Ganadería }\end{array}$ & Subóptima \\
\hline $\mathrm{ME}$ & Léntico & 115 & $\begin{array}{l}\text { Sitio Ramsar } \\
\text { no. } 2045 \text { y Área } \\
\text { Natural } \\
\text { Protegida } \\
\text { (ZSCE) } \\
\end{array}$ & 2.947 & $\begin{array}{l}\text {-Vivienda } \\
\text {-Humedal artificial }\end{array}$ & Óptima \\
\hline LK & Léntico & 110 & $\begin{array}{l}\text { Sitio Ramsar } \\
\text { no. } 1787 \text { y Área } \\
\text { Natural } \\
\text { Protegida } \\
\text { (ZSCE) }\end{array}$ & 2.762 & $\begin{array}{l}\text {-Contaminación } \\
\text { significativa por } \\
\text { residuos sólidos } \\
\text {-Infraestructura } \\
\text { hidráulica del } \\
\text { Sistema de Agua } \\
\text { Potable y } \\
\text { Alcantarillado } \\
\text { Municipal } \\
\text { (SAPAM) } \\
\text {-Vivienda } \\
\end{array}$ & Regular \\
\hline$C M$ & Léntico & 16 & & 2.115 & $\begin{array}{l}\text {-Contaminación } \\
\text { significativa por } \\
\text { residuos sólidos } \\
\text {-Relleno de la } \\
\text { superficie } \\
\text { inundable } \\
\text {-Ganadería } \\
\text {-Incendios (60\% } \\
\text { del área) } \\
\text {-Vivienda }\end{array}$ & Pobre \\
\hline
\end{tabular}

\section{Odonatofauna de los humedales de SCLC}

Un total de 1,784 individuos (966 recolectados, 818 marcados y liberados) pertenecientes a cinco familias, diez géneros y 14 especies fueron registrados (Cuadro 2). Las familias con la mayor riqueza fueron Coenagrionidae (tres géneros y seis especies) y Libellulidae (tres géneros y cuatro especies), así como el género Argia (tres especies). Con una especie cada una, la menor riqueza se registró en las familias Calopterygidae y Lestidae. La representatividad del muestreo correspondió 
al $82.35 \%$ del total de especies (17) que potencialmente se distribuyen en el área de estudio (Fig. 2).

Cuadro 2. Lista de especies de Odonata y su distribución $(x)$ en cuatro humedales de montaña de San Cristóbal.

\begin{tabular}{|c|c|c|c|c|c|c|}
\hline \multirow[t]{2}{*}{ Familia } & \multirow[t]{2}{*}{ Género } & \multirow[t]{2}{*}{ Especie } & \multicolumn{4}{|c|}{ Humedales } \\
\hline & & & $\mathrm{LC}$ & $\mathrm{ME}$ & LK & $\mathrm{CM}$ \\
\hline Calopterygidae & Hetaerina & H. vulnerata & $x$ & $x$ & & \\
\hline \multirow[t]{6}{*}{ Coenagrionidae } & Argia & A. anceps & $x$ & & & \\
\hline & & A. chelata & $x$ & & & \\
\hline & & A. funebris & $x$ & $x$ & $x$ & $x$ \\
\hline & Enallagma & E. rua & $\mathrm{x}$ & $x$ & $\mathrm{x}$ & $x$ \\
\hline & Ischnura & I. denticollis & $x$ & $x$ & $x$ & $x$ \\
\hline & & 1. ramburii & & & $x$ & \\
\hline Lestidae & Lestes & L. alacer & $x$ & $x$ & $x$ & $x$ \\
\hline \multirow[t]{2}{*}{ Aeshnidae } & Oplonaeschna & O. armata & $x$ & & & \\
\hline & Rhionaeschna & R. jalapensis & $x$ & $x$ & $x$ & $x$ \\
\hline \multirow[t]{4}{*}{ Libellulidae } & Libellula & L. foliata & & $x$ & & \\
\hline & Macrothemis & M. inacuta & & & $x$ & \\
\hline & & M. pseudimitans & $\mathrm{x}$ & & & \\
\hline & Sympetrum & S. illotum & $\mathrm{x}$ & $x$ & $x$ & $x$ \\
\hline
\end{tabular}

\section{Diversidad local}

Aunque no se encontraron diferencias estadísticas significativas en la riqueza de Odonata entre los diferentes humedales ( $\mathrm{H}=3.508 ; p=0.319)$, LC obtuvo el mayor número de especies (Fig. 3 ). Así mismo, se hallaron diferencias significativas en la abundancia de Odonata entre los diferentes sitios ( $F=8.296$ y $p<0.01$ ), en este caso ME y LK obtuvieron el mayor registro de odonatos. Por otro lado, LC y LK fueron los humedales con la mayor diversidad de odonatos (área= 5.26 y 3.97, respectivamente), mientras que CM y ME fueron menos diversos (área $=3.68$ y 3.32, correspondientemente).

Las especies dominantes en el área de estudio fueron Ischnura denticollis Burmeister, 1839 (42.61\%) y Enallagma rua Donnelly, 1968 (31.61\%), además de otras especies numéricamente importantes (Argia funebris Hagen, 1861; Lestes alacer Hagen, 1861; Rhionaeschna jalapensis Williamson, 1908; Sympetrum illotum Hagen, 1861) que en conjunto representan el $21.5 \%$ de la abundancia total. 


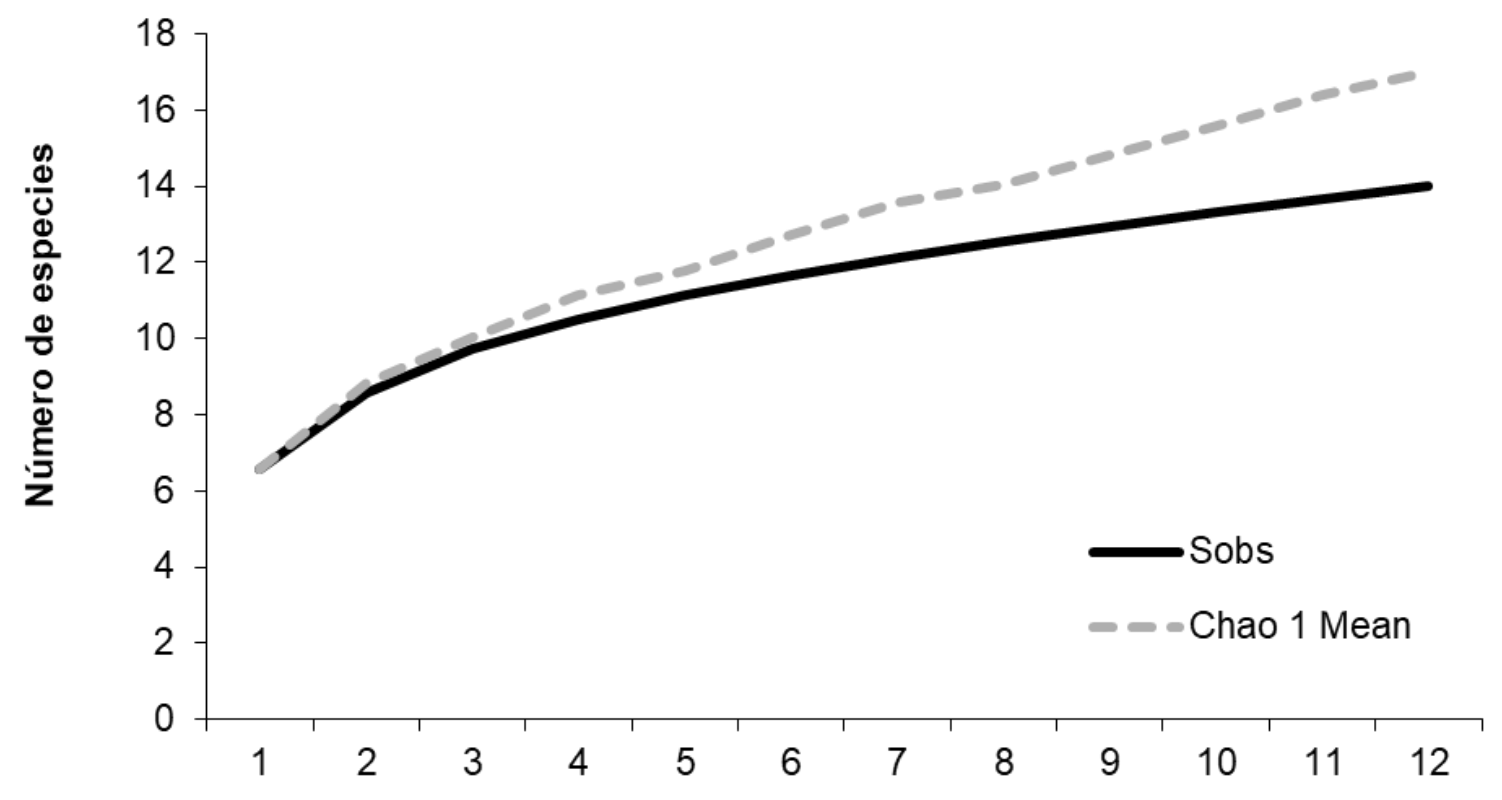

\section{Meses de muestreo}

Figura 2. Curva de acumulación de especies de Odonata en cuatro humedales de montaña de San Cristóbal de las Casas, Chiapas, México.

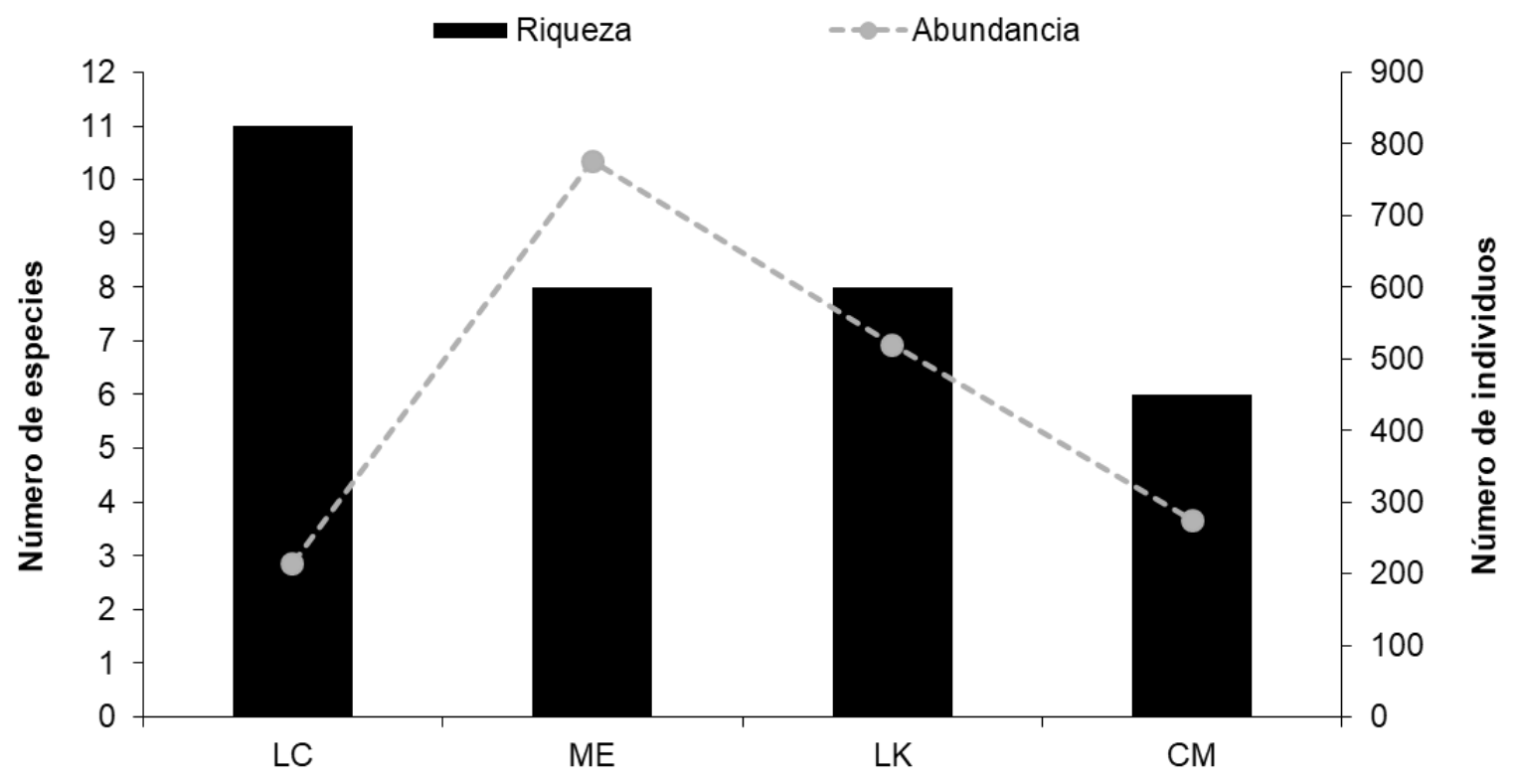

Figura 3. Relación entre la riqueza y abundancia de Odonata en los cuatro diferentes humedales en San Cristóbal de las Casas, Chiapas, México. 


\section{Diversidad beta}

Los imagos de Hetaerina vulnerata Hagen en Selys, 1853 y Libellula foliata Kirby, 1869 reposaban sobre la vegetación de un arroyo próximo a la laguna que integra el humedal ME, por lo tanto, estas dos especies y las que se distribuyen exclusivamente en LC (Argia anceps Garrison, 1996; Argia chelata Calvert, 1901; Macrothemis pseudimitans Calvert, 1898; Oplonaeschna armata Hagen, 1861), integran el $42.85 \%$ de especies limitadas a ambientes lóticos; mientras que Ischnura ramburii Selys, 1850 y Macrothemis inacuta Calvert, 1898, integran el 14.28\% de especies restringidas a ambientes lénticos. El resto de las especies (A. funebris, E. rua, I. denticollis, L. alacer, $R$. jalapensis y S. illotum) representan el $42.85 \%$ de los odonatos distribuidos en ambos tipos de ambiente.

La mayor similitud faunística se encontró entre ME y LK $\left(\mathrm{I}_{\mathrm{M}-\mathrm{H}}=0.97\right)$, mientras que la menor se encontró entre LC y ME ( $\left.\mathrm{I}_{\mathrm{M}-\mathrm{H}}=0.15\right)$. Debido a ello, el dendrograma separa la fauna de odonatos de LC del resto de humedales (Fig. $4 ; I_{M-H} \leq 0.45$ ); a su vez, la odonatofauna de CM se separa de ME y LK ( $\left.I_{\mathrm{M}-\mathrm{H}} \leq 0.93\right)$, por lo que la fauna de Odonata de estos dos últimos humedales está estrechamente relacionada.

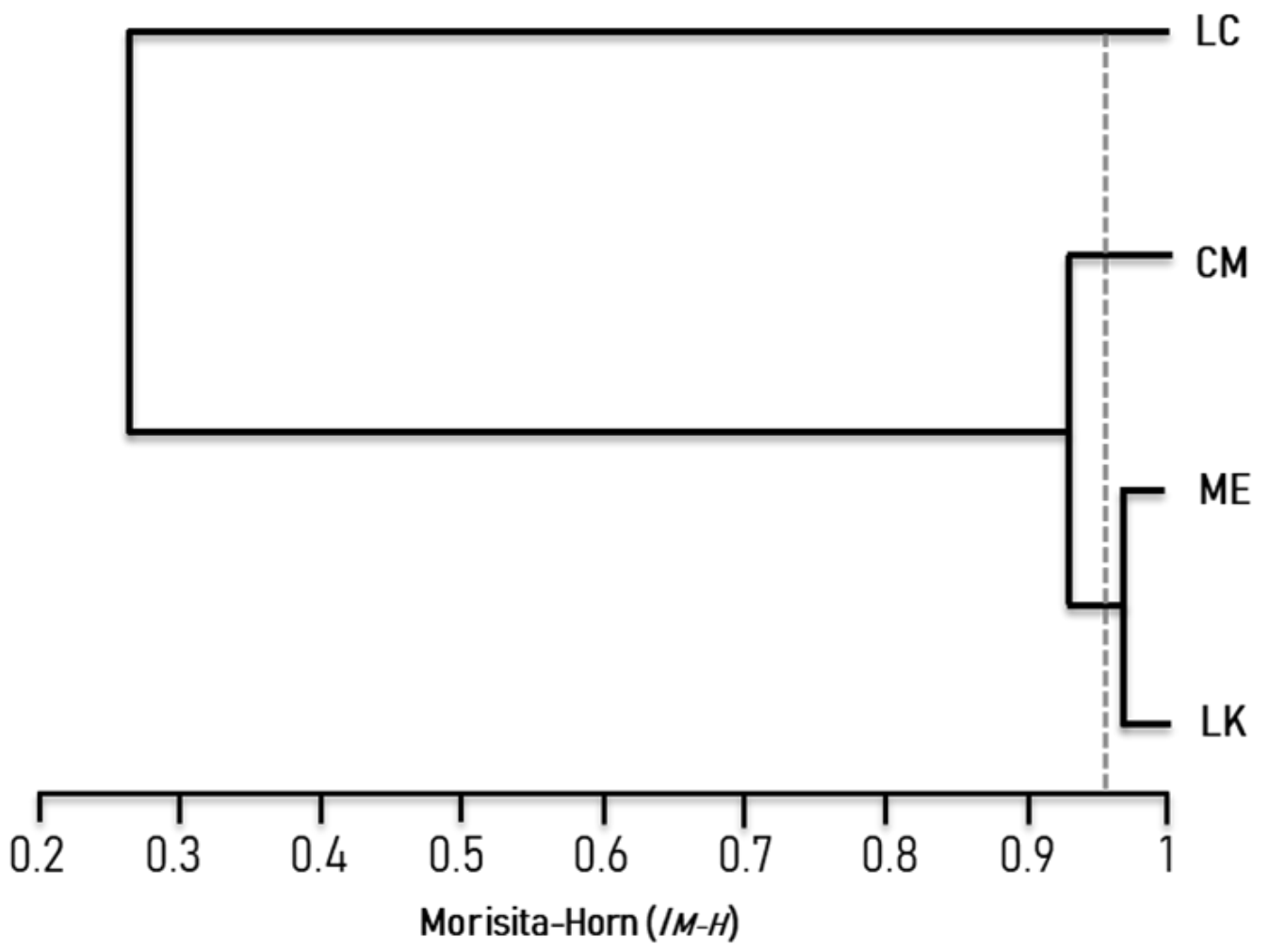

Figura 4. Dendrograma de similitud $\left(\mathrm{I}_{\mathrm{M}-\mathrm{H}}\right)$ odonatofaunística entre los cuatro diferentes humedales de montaña en San Cristóbal de las Casas, Chiapas, México.

\section{DISCUSIÓN}

Odonatofauna de los humedales de SCLC. Se presenta el primer estudio sistemático de la odonatofauna para los humedales de SCLC, que constituye el $7.48 \%$ del total de especies 
López-Díaz et al.: Odonata como indicador de la calidad ambiental de humedales de montaña

registradas para Chiapas (González-Soriano \& Paulson, 2011; Gómez-Tolosa et al., 2015), así como el 3.93\% de las especies reconocidas en México (Cuevas-Yañez et al., 2017b). Nueve de las 11 especies documentadas en la región por González-Soriano y Paulson (2011) se reportan en este trabajo, y se complementa con cinco registros más para el área de estudio ( $A$. anceps, I. ramburii, L. alacer, M. inacuta y M. pseudimitans), incrementando a 16 el total de especies para SCLC. Aunque Erythrodiplax abjecta Rambur, 1842 y Rhionaeschna cornigera Brauer, 1865 no se reportan en este estudio, son especies que podrían completar la lista de la odonatofauna en la región. Rhionaeschna cornigera parece ser una especie rara en SCLC, ya que ha sido encontrada previamente en ambientes lóticos y lénticos en zonas montañosas, y en el estado de Chiapas en las Lagunas de Montebello (De Marmels, 1982; E. González-Soriano obs. pers., datos no publicados). Las especies del género Erythrodiplax son comunes en ambientes lénticos (Cuevas-Yáñez et al., 2017a) y pueden aprovechar las modificaciones provocadas por la urbanización (Monteiro-Júnior et al., 2015), no obstante, un sesgo en el muestreo seguramente repercutió en el registro de E. abjecta en el área de estudio, considerando que los anisópteros son más difíciles de capturar como adultos que los caballitos del diablo (Corbet, 1999).

Diversidad local. El tipo de ambiente lótico que representa LC probablemente explica su mayor riqueza y diversidad de odonatos. A diferencia de los cuerpos de agua lénticos, los ambientes lóticos se caracterizan por tener una mayor heterogeneidad de sustratos y microhábitats disponibles para las larvas de Odonata (Stewart \& Samways, 1998; Balzan, 2012). Diversos autores (Oppel, 2005; Novelo-Gutiérrez \& Gómez-Anaya, 2009; De Paiva et al., 2010; Monteiro-Júnior et al., 2015; Calvão et al., 2020) han documentado que la eliminación de la vegetación ribereña indica una pérdida en la integridad del hábitat, favoreciendo la presencia de especies oportunistas, quienes aprovechan los hábitats lénticos expuestos y aislados, incrementando la riqueza de odonatos. La ganadería en LC probablemente favoreció la presencia de algunas especies como $L$. alacer, quien obtuvo la mayor abundancia en este humedal y que ha sido reportada en aguas estancadas (González-Soriano \& Novelo-Gutiérrez, 1991).

En los ambientes lénticos, la mayor riqueza de odonatos encontrada en ME y LK posiblemente se atribuye a la gran diversidad de macrófitas (Benazzouz et al., 2009), que para el caso de ME está integrada por las enraizadas emergentes Schoenoplectus americanus (Pers.) Volkart ex Schinz \& R. Keller, Schoenoplectus tabernaemontani (C.C. Gmel.) Palla, Typha domingensis Pers., Typha latifolia L., Juncus effusus L., y por la enraizada subacuática Potamogeton illinoensis Morong; mientras que en LK se distribuyen las enraizadas emergentes Eleocharis elongata Chapm., S. californicus, T. latifolia, y por la flotante E. crassipes. Por lo tanto, la riqueza de odonatos es favorecida por aquellos cuerpos de agua cuya vegetación es de estructura mixta, a diferencia de las masas densas monotípicas (Mabry \& Dettman, 2010), como se observó en CM, humedal con la menor riqueza de odonatos y cuya vegetación acuática está dominada por la enraizada emergente $S$. californicus, y con menor abundancia se distribuyen Cyperus lundellii O’Neil, Eleocharis filiculmis Kunth, así como la enraizada sumergida Ranunculus geoides H.B.K.

ME y LK son humedales con calidad visual óptima y regular, respectivamente, con mayor abundancia de odonatos asociada, probablemente, a la conservación de su vegetación acuática. Por otra parte, LC y CM son humedales con calidad visual subóptima y pobre, respectivamente, en 
donde posiblemente el pisoteo y forrajeo de las macrófitas por el pastoreo de ganado, además de los incendios intencionales en el caso de $\mathrm{CM}$, repercutieron en una baja abundancia de odonatos. Si bien la relevancia que tiene este tipo de vegetación en el ciclo de vida de los Odonata ha sido ampliamente reconocida (Westfall \& Tenessen, 1996; Stewart \& Samways, 1998; Balzan, 2012; Patra et al., 2012), nuestros resultados sugieren que la abundancia de odonatos se vio afectada por la estructura física de las especies vegetales más abundantes (Hornung \& Rice, 2003; Foote $\&$ Rice, 2005), asociada con la disponibilidad de sitios de oviposición y perchas para los adultos de Odonata (Corbet, 1999; Brito et al., 2020). Por lo tanto, como uno de los parámetros evaluados en la calidad visual, el grado de alteración de la vegetación acuática fue más importante en las diferencias encontradas de la abundancia y diversidad de odonatos en los cuatro humedales, ya que las características del sustrato son determinantes para la presencia de cada especie y la limitación de su abundancia (Juen et al., 2007), principalmente para I. denticollis y $E$. rua, especies más abundantes en los tres humedales lénticos.

Córdoba-Aguilar (1993) ha descrito a I. denticollis como una especie con alta densidad poblacional y fidelidad dentro de los estanques, relacionada con la estructura de la vegetación acuática flotante y abundante (Gómez-Anaya \& Novelo-Gutiérrez, 2015). En este estudio, observamos a $E$. rua con una alta abundancia relativa (aunque menor en comparación a la de $I$. denticollis) entre la vegetación emergente y la flotante, que también se ha observado en otras especies del género Enallagma (véase Foote \& Rice, 2005). Una explicación plausible de la asociación benéfica de I. denticollis y $E$. rua con las macrófitas podría ser su oviposición endofítica obligada, ya que las hembras de ambas especies requieren de diferentes sustratos para realizar esta actividad. Además, la vegetación acuática podría favorecer a I. ramburii, L. alacer y $R$. jalapensis, especies con este mismo tipo de oviposición (González-Soriano \& Novelo-Gutiérrez, 1991; von Ellenrieder, 2003; Upson et al., 2007), las dos últimas con alta abundancia en este estudio.

Diversidad beta. La estructura del paisaje puede afectar las ocurrencias de las libélulas adultas quienes buscan hábitats adecuados para desarrollarse (Corbet, 1999). Taniguchi y Tokeshi (2004) refieren que la diversidad de la vegetación acuática puede incrementar la complejidad de hábitat (i.e. número de morfologías y número de diferentes estructuras de un hábitat). ME y LK son humedales con agua permanente, donde su mayor diversidad de macrófitas, integrada principalmente por especies enraizadas emergentes y en menor grado por enraizadas flotantes, probablemente influyó en una mayor similitud de odonatos entre ellos, ya que las distintas formas de vida de la vegetación acuática repercuten de manera importante en las especies de Odonata en los sistemas lagunares (Carchini et al., 2005; Balzan, 2012). Por ejemplo, I. ramburii es un caballito del diablo que suele habitar en aguas con corriente lenta y con vegetación densa y expuesta a la luz solar (Upson et al., 2007) como se observó en LK; mientras que L. foliata fue observada sobre la vegetación emergente de un arroyo cercano al humedal ME. En ambas especies, la vegetación acuática podría ser un factor que refleja la heterogeneidad del hábitat (Chovanec \& Waringer, 2001) y, su distribución exclusivamente en cada uno de estos humedales, podría relacionarse con distintos patrones de diferenciación de nichos (Chovanec et al., 2005). Así mismo, el área de la superficie de cuerpos de agua lénticos, como un requisito de las etapas larvarias, puede ser importante en la determinación de la composición de especies de odonatos 
adultos (Kadoya et al., 2008). Por lo tanto, la extensión similar de ME y LK (SEMAHN, 2010, 2011) probablemente repercutió en su similitud, especialmente al encontrar la misma riqueza de libélulas en estos dos cuerpos de agua.

La configuración del paisaje permite predecir las probabilidades de ocupación, extinción y colonización de odonatos (Harms et al., 2014). Worthen y Chamlee (2020) refieren que las diferencias en las tolerancias de las especies a los impactos antropogénicos pueden causar que ensambles originalmente heterogéneos de libélulas, tolerantes e intolerantes, se "descompongan" a ensambles más pequeños de especies más tolerantes a lo largo del tiempo y/o espacio, creando subconjuntos anidados, como podría estar ocurriendo con A. funebris, E. rua, I. denticollis, L. alacer, $R$. jalapensis y $S$. illotum, especies muy abundantes y con distribución en los cuatro humedales. Los requisitos de hábitat menos específicos (De Paiva et al., 2010) y la alta capacidad de dispersión (Gómez-Anaya \& Novelo-Gutiérrez, 2015) de estas especies pueden explicar su distribución en ambientes lóticos y lénticos. Además, la conectividad del paisaje y la distancia entre estanques puede influir en la probabilidad de dispersión de odonatos, principalmente en las especies más pequeñas (Harms et al., 2014), como podría ocurrir con E. rua e l. denticollis, especies dominantes en este estudio.

LC es un humedal con disponibilidad temporal de agua asociada a los meses de precipitación, en donde la velocidad de corriente probablemente es uno de los requerimientos específicos del hábitat (McPeek, 2008) para especies como H. vulnerata, A. anceps, A. chelata, $M$. pseudimitans y $O$. armata, distribuidas exclusivamente en ambientes lóticos. La velocidad de corriente favorece una mayor cantidad de oxígeno disuelto e influye en la temperatura del agua, así como en los cambios continuos del sustrato (Merrit \& Cummins, 1996). Por otro lado, la composición de especies de macrófitas en LC es distinta a la del resto de los humedales, conformada por las enraizadas emergentes Mimulus glabratus Kunth, Polygonum punctatum Elliott, Berula erecta (Huds.) Coville, y por la flotante libre Cardamine bonariensis Pers. GómezAnaya y Novelo-Gutiérrez (2015) describen que las diferencias en la composición vegetal y la dominancia de algunas especies pueden ser determinantes en la presencia de ciertos odonatos, al representar focos de actividad territorial por la disponibilidad de sustratos para la oviposición (Corbet, 1999), como se observó en $\mathrm{H}$. vulnerata. La mayor disimilitud de la odonatofauna entre LC y ME podría explicarse por estas diferencias en la permanencia del agua, la velocidad de corriente y la sombra, que permiten distinguir a los ensambles de libélulas y caballitos del diablo (Oppel, 2005; De Paiva et al., 2010).

La actual fragmentación de los humedales de montaña en SCLC (Cruz \& Hernández, 2010) posiblemente conllevará a que especies raras como $A$. anceps, $A$. chelata, I. ramburii, L. foliata y $O$. armata corran el riesgo de extinguirse al ser susceptibles a los cambios ambientales inducidos por las actividades humanas (Chovanec \& Waringer, 2001; Gómez-Anaya \& Novelo-Gutiérrez, 2010), especialmente aquellas con fase pre-reproductiva larga, quienes dependen fuertemente de la estructura del paisaje al utilizar hábitats acuáticos y terrestres por un tiempo prolongado (Kadoya et al., 2008). Por lo tanto, los dos tipos de sistema hidrológico (lótico/léntico) en los humedales de SCLC, además del ambiente terrestre circundante, son importantes para la conservación de estas especies de Odonata en cuerpos de agua urbanos y de alta montaña. 
Agradecimientos. Al Dr. Rosser W. Garrison (Office of the Los Angeles County Agricultural Commissioner-U.S.A.), por su invaluable apoyo en la determinación taxonómica del género Argia. Al Dr. Mario Ishiki Ishihara y al Sr. Miguel Martínez Icó (Herbario de El Colegio de la Frontera Sur, unidad San Cristóbal), por su apoyo en la identificación de especies de macrófitas. A la Lic. Ma. Esther Álvarez Rodríguez, por su apoyo en el trabajo de campo. A los revisores anónimos por sus acertadas observaciones al documento.

\section{LITERATURA CITADA}

Barbour, M., Gerritsen, J., Snyder, B., Stribling, J. (1999) Rapid bioassessment protocols for use in streams and wadeable rivers: periphyton, benthic macroinvertebrates and fish. Environmental Protection Agency, Office of Water. Washington D.C., United States, 343 pp.

Balzan, M. V. (2012) Associations of dragonflies (Odonata) to habitat variables within the Maltese Islands: a spatio-temporal approach. Journal of Insect Science, 12 (87), 1-18. https://doi.org/10.1673/031.012.8701

Benazzouz, B., Mouna, M., Amezian, M., Bensusan, K., Pérez, C., Cortés, J. (2009) Assessment and conservation of the dragonflies and damselflies (Insecta: Odonata) at the marshes of Smir. Bulletin de l'Institut Scientifique, 31, 79-84.

Boix, D., Caiola, N., Cañedo-Argüelles, M., Gascón, S., Ibáñez, C., Nebra, A., Quintana, X., Rieradevall, M., Sala, J., Sánchez-Millaruelo, N., Solá, C., Munné, A. (2010) Avaluació de l'estat ecològic de les zones humides $i$ ajust dels indicadors de qualitat; Índexs QAELS 2010, ECELS i EQAT. Agència Catalana de l'Aigua, Departament de Medi Ambient i Habitatge, Generalitat de Catalunya. Barcelona, España, 209 pp.

Brito, J. S., Michelan, T. S., Juen, L. (2020) Aquatic macrophytes are important substrates for Libellulidae (Odonata) larvae and adults. Limnology, 22 (3), 139-149. https://doi.org/10.1007/s10201-020-00643-x

Bulánková, E. (1997) Dragonflies (Odonata) as bioindicators of environment quality. Biologia, Bratislava, 52 (2), 177-180.

Calvão, L. B., de Paiva, C. K. S., Brito, J., Fares, A. L., Maia, C., Michelan, T. S., de Assis Montag, L. F., Juen, L. (2020) Influence of biotic and abiotic factors on adult Odonata (Insecta) in Amazon streams. Animal Biology, 71, 1-18. https://doi.org/10.1163/15707563-bja10047

Carchini, G., Solimini, A. G., Ruggiero, A. (2005) Habitat characteristics and odonate diversity in mountain ponds of central Italy. Aquatic Conservation Marine and Freshwater Ecosystems, 15 (6), 573-581.

https://doi.org/10.1002/aqc.741

Catling, P. (2005) A potential for the use of dragonfly (Odonata) diversity as a bioindicator of the efficiency of sewage lagoons. Canadian Field-Naturalist, 119 (2), 233-236. https://dx.doi.org/10.22621/cfn.v119i2.111

Chovanec, A., Waringer, J. (2001) Ecological integrity of river-floodplain systems-assessment by dragonfly surveys (Insecta: Odonata). Regulated Rivers: Research \& Management, 17, 493507.

https://doi.org/10.1002/rrr.664 
Chovanec, A., Waringer, J. (2005) Dragonflies (Insecta: Odonata) as indicators of the ecological health of wetland ecosystems. Internationale Vereinigung für theoretische und angewandte Limnologie: Verhandlungen, 29, 422-425.

https://doi.org/10.1080/03680770.2005.11902047

Colwell, R. (2004) Estimates: statistical estimation of species richness and shared species from samples, version 7.5. Available at: http://viceroy.eeb.uconn.edu/estimates/ (accessed on February 20, 2015).

Corbet, P. S. (1999) Dragonflies: Behavior and Ecology of Odonata. Comstock Publishing Associates. Colchester, United Kingdom, 829 pp.

Córdoba-Aguilar, A. (1993) Population structure in Ischnura denticollis (Burmeister) (Zygoptera: Coenagrionidae). Odonatologica, 22 (4), 455-464.

Crisci, J. V., López, M. F. (1983) Introducción a la teoría y práctica de la Taxonomía Numérica. Monografías de la OEA (Serie Biología). Washington, United States, 132 pp.

Cruz, J., Hernández, F. (2010) Los humedales de San Cristóbal de Las Casas, Chiapas: actores y disputas. Revista de Geografía Agrícola, 44, 91-104.

Cuevas-Yañez, K. (2007) Los odonatos (Insecta: Odonata) de la Hidroeléctrica de Patla (El Pozo) y del río Tecpatlán, Zihuateutla, Puebla, México. Dugesiana, 14 (2), 83-91.

Cuevas-Yañez, K., Benítez, M., Rocha, M., Córdoba-Aguilar, A. (2017a) Large-scale human environmental intervention is related to a richness reduction in Mexican odonates. Revista Mexicana de Biodiversidad, 88 (3), 664-673.

https://dx.doi.org/10.1016/j.rmb.2017.06.008

Cuevas-Yañez, K., Espinosa-Rivera, J. C., Martínez-Falcón, A. P., Córdoba-Aguilar, A. (2017b) Are all Mexican odonate species documented? An assessment of species richness. Systematics and Biodiversity, 15 (3), 253-258. https://dx.doi.org/10.1080/14772000.2016.1249529

De Marmels, J. (1982) Dos náyades nuevas de la familia Aeshnidae (Odonata: Anisoptera). Boletín de Entomología de Venezuela Nueva Serie, 2 (12), 102-106.

De Paiva, D., De Marco, P., Chaves, D. (2010) Adult odonate abundance and community assemblage measures as indicators of stream ecological integrity: A case study. Ecological Indicators, 10, 744-752.

https://doi.org/10.1016/j.ecolind.2009.12.004

Di Battista, T., Fortuna, F., Maturo, F. (2016) Environmental monitoring through functional biodiversity tools. Ecological Indicators, 60, 237-247.

http://dx.doi.org/10.1016/j.ecolind.2015.05.056

Di Battista, T., Fortuna, F., Maturo, F. (2017) BioFTF: An R package for biodiversity assessment with the functional data analysis approach. Ecological Indicators, 73, 726-732.

https://dx.doi.org/10.1016/j.ecolind.2016.10.032

Foote, A. L., Rice, C. L. (2005) Odonates as biological indicators of grazing effects on Canadian prairie wetlands. Ecological Entomology, 30 (3), 273-283.

https://doi.org/10.1111/j.0307-6946.2005.00701.x

Gómez-Anaya, J. A., Novelo-Gutiérrez, R. (2010) Richness and structure of an Odonata larval assemblage from Río Pinolapa, Tepalcatepec, Michoacán, Mexico in relation to characteristics their habitat. Odonatologica, 39 (4), 287-303. 
Gómez-Anaya, J. A., Novelo-Gutiérrez, R. (2015) A case of successful restoration of a tropical wetland evaluated through its Odonata (Insecta) larval assemblage. Revista de Biología Tropical, 63 (4), 1043-1058.

https://doi.org/10.15517/rbt.v63i4.15738

Gómez-Tolosa, M. L., Mendoza-Cuenca, L. F., Rioja-Paradela, T. M., Espinoza-Medinilla, E. E., Alonso-Eguía-Lis, P. E., Rivera-Velázquez, G., Penagos-García, F. E., Pérez-Munguía, R. M., Ortega-Salas, H., Gómez-Cristiani, M., Gómez-Gutiérrez, R. B. (2015) Odonata (Insecta) de tres cuencas en la costa de Chiapas: lista de especies y registro nuevo. Revista Mexicana de Biodiversidad, 86, 1-7. https://dx.doi.org/10.7550/rmb.48665

González-Soriano, E., Novelo-Gutiérrez, R. (1991) Odonata de la Reserva de la Biósfera de La Michilía, Durango, México: Parte I, imagos. Folia Entomológica Mexicana, 81, 67-105.

González-Soriano, E., Paulson, D. R. (2011) Odonata de Chiapas. Pp. 299-314. In: F. ÁlvarezNoguera (Ed.). Chiapas, estudios sobre su biodiversidad biológica. Instituto de Biología, UNAM, México.

Guisande, C., Heine, J., García-Roselló, E., González-Dacosta, J., Vilas, L. G., Perez-Schofield, B. J. G. (2017) DER: An algorithm for comparing species diversity between assemblages. Ecological Indicators, 81, 41-46. https://doi.org/10.1016/j.ecolind.2017.05.049

Hammer, O., Harper, D., Ryan, P. (2001) PAST: Paleontological Statics Software Package for Education and Data Analysis. Palaeontologia Electronica, 4, 1-9.

Harms, T. M., Kinkead, K. E., Dinsmore, S. J. (2014) Evaluating the effects of landscape configuration on site occupancy and movement dynamics of odonates in lowa. Journal of Insect Conservation, 18 (3), 307-315. https://dx.doi.org/10.1007/s10841-014-9638-4

Holland, C., Homea, J., Gwin, S., Kentula, M. (1995) Wetland degradation and loss in the rapidly urbanizing area of Portland, Oregon. Wetlands, 15 (4), 336-345.

https://dx.doi.org/10.1007/BF03160888

Hornung, J. P., Rice, C. L. (2003) Odonata and wetland quality in southern Alberta Canada: a preliminary study. Odonatologica, 32 (2), 119-129.

Juen, L., Cabette, H. S. R., De Marco, P. (2007) Odonate assemblage structure in relation to basin and aquatic hábitat structure in Pantanal wetlands. Hydrobiologia, 579, 125-134.

https://doi.org/10.1007/s10750-006-0395-6

Kadoya, T., Suda, S., Tsubaki, Y., Washitani, I. (2008) The sensitivity of dragonflies to landscape structure differs between life-history groups. Landscape Ecology, 23 (2), 149-158. https://dx.doi.org/10.1007/s10980-007-9151-1

Kutcher, T. E., Bried, J. T. (2014) Adult Odonata conservatism as an indicator of freshwater wetland condition. Ecological Indicators, 38, 31-39. https://dx.doi.org/10.1016/j.ecolind.2013.10.028

Mabry, C., Dettman, C. (2010) Odonata Richness and Abundance in Relation to Vegetation Structure in Restored and Native Wetlands of the Prairie Pothole Region, USA. Ecological Restoration, 28 (4), 475-484.

https://dx.doi.org/10.3368/er.28.4.475 
López-Díaz et al.: Odonata como indicador de la calidad ambiental de humedales de montaña

McDonell, M., Pickett, S. (1990) Ecosystem structure and function along urban-rural gradients: an unexploited opportunity for ecology. Ecology, 71 (4), 1232-1237. https://dx.doi.org/10.2307/1938259

McPeek, M. A. (2008) Ecological factors limiting the distributions and abundances of Odonata. Pp. 51-62. In: A. Córdoba-Aguilar (Ed.). Dragonflies and Damselflies: model organisms for ecological and evolutionary research. Oxford, University Press, New York, United States.

Merrit, R., Cummins, K. (1996) An introduction to the aquatic insects of North America. KendallHunt Publishing Company. lowa, Estados Unidos, 862 pp.

Monteiro-Júnior, C. S., Juen, L., Hamada, N. (2015) Analysis of urban impacts on aquatic hábitats in the central Amazon basin: adults odonates as bioindicators of environmental quality. Ecological Indicators, 48, 303-311.

https://dx.doi.org/10.1016/j.ecolind.2014.08.021

Morse, T. (1998) Catching and preserving dragonflies FAQ. Available at: https://www.afn.org/iori/morse2.html (accessed on April 23, 2016).

Novelo-Gutiérrez, R., Gómez-Anaya, J. A. (2009) A comparative study of Odonata (Insecta) assemblages along an altitudinal gradient in the sierra de Coalcomán Mountains, Michoacán, Mexico. Biodiversity Conservation, 18 (3), 679-698.

https://doi.org/10.1007/s10531-008-9533-y

Oppel, S. (2005) Habitat associations of an Odonata community in a lower montane rainforest in Papua New Guinea. International Journal of Odonatology, 8 (2), 243-257.

https://dx.doi.org/10.1080/13887890.2005.9748256

Palacino-Rodríguez, F., Contreras-Sánchez, N. A. (2014) Does experimental marking of wings influence resighting success in Mesamphiagrion laterale and Erythrodiplax umbrata? (Odonata: Coenagrionidae, Libellulidae). Odonatologica, 43 (3), 237-246.

Patra, A., Santra, K. B., Manna, C. K. (2012) Macroinvertebrate community associated with macrophytes in the Santragachi Jheel Lake, West Bengal, India. Ekológica (Bratislava), 31 (3), 274-294. https://doi.org/10.4149/ekol_2012_03_274

Ramírez, A. (2010) Odonata. Revista de Biología tropical, 58 (4), 97-136.

R Core Team (2013) R: A Language and Environment for Statistical Computing. R Foundation for Statistical Computing, Vienna, Austria. Available at: http://www.R-project.org/ (accessed on July 28, 2019).

Schorr, M., Paulson, D. (2019) World Odonata List. Available at: https://www.pugetsound.edu/academics/academic-resources/slatermuseum/biodiversityresources/dragonflies/world-odonata-list2/ (accessed on July 18, 2019).

Secretaria de Medio Ambiente, Vivienda e Historia Natural (SEMAHN) (2010) Programa de manejo de la zona sujeta a conservación ecológica "Humedales de montaña La Kisst". Gobierno del Estado de Chiapas. Chiapas, México, 120 pp.

Secretaría de Medio Ambiente, Vivienda e Historia Natural (SEMAHN) (2011) Programa de manejo de la zona sujeta a conservación ecológica "Humedales de montaña María Eugenia". Gobierno del Estado de Chiapas. Chiapas, México, 107 pp.

StatSoft (1991) CSS: Statistica handbook, vol. II. Statsoft Inc. Oklahoma, United States, 104 pp.

Stewart, D. A., Samways, M. J. (1998) Conserving dragonfly (Odonata) assemblages relative to river dynamics in an African savanna game reserve. Conservation Biology, 12 (3), 683-692. 
https://dx.doi.org/10.1111/j.1523-1739.1998.96465.x

Stewart, R. E, Kantrud, H. A. (1971) Classification of natural ponds and lakes in the glaciated prairie region. Ed. Bureau of Sport Fisheries and Wildlife. Washington, United States, 57 pp.

Taniguchi, H., Tokeshi, M. (2004) Effects of habitat complexity on benthic assemblages in a variable environment. Freshwater Biology, 49 (9), 1164-1178. https://doi.org/10.1111/j.1365-2427.2004.01257.x

Tuomisto, H. (2010) A diversity of beta diversities: Straightening up a concept gone awry, part 2. Quantifying beta diversity and related phenomena. Ecography, 33, 23-45. https://doi.org/10.1111/j.1600-0587.2009.06148.x

Upson, S., Danforth, D., González-Soriano, E., Behrstock, R. A., Bailowitz, R. A. (2007) A preliminary checklist of the Odonata of Sonora, Mexico. Bulletin of American Odonatology, 10 (2), 23-54.

Vásquez, M. A. (2007) Documento preparado para la elaboración participativa del diagnóstico y plan de manejo de los humedales de San Cristóbal de la Casas, Chiapas. El Colegio de La Frontera Sur. Chiapas, México, 25 pp.

von Ellenrieder, N. (2003) A synopsis of the Neotropical species of 'Aeshna' Fabricius: The genus Rhionaeschna Förster (Odonata: Aeshnidae). Tijdschrift voor Entomologie, 146, 67-207. https://dx.doi.org/10.1163/22119434-900000120

Westfall, M. J., Tenessen, K. (1996) Odonata. Pp. 164-211. In: K. Cummins, R. Merritt (Eds.). An introduction aquatic insects of North America. Dubuque: Kendall/Hunt Publishing Company. California, United States.

Woodward, R. T., Wui, Y. S. (2001) The economic value of wetland services: a meta-analysis. Ecological Economics, 37 (2), 257-270. https://doi.org/10.1016/S0921-8009(00)00276-7

Worthen, W. B., Chamlee, M. G. (2020) Determinants of adult odonate community structure at several spatial scales: effects of habitat type and landscape context. International Journal of Odonatology, 23 (4), 365-379.

https://doi.org/10.1080/13887890.2020.1796831 